\title{
Effects of carvedilol on left ventricular function, mass, and scintigraphic findings in isolated left ventricular non-compaction
}

\author{
M Toyono, C Kondo, Y Nakajima, M Nakazawa, K Momma, K Kusakabe
}

\begin{abstract}
A four month old infant with isolated left ventricular non-compaction was treated with carvedilol. Haemodynamic studies and various types of imaging-including echocardiography, radiographic angiography, magnetic resonance imaging, and single photon emission computed tomography with ${ }^{201} \mathbf{T} 1,{ }^{123} \mathbf{I}-\beta$-methyliodophenylpentadecanoic acid (BMIPP), and ${ }^{123}$ I-metaiodobenzylguanidine (MIBG)were performed before and 14 months after treatment. Left ventricular ejection fraction increased from $30 \%$ to $57 \%$, and left ventricular end diastolic volume, end systolic volume, and end diastolic pressure showed striking reductions during treatment. Left ventricular mass decreased to about two thirds of the baseline value after treatment. Per cent wall thickening increased after carvedilol in the segments corresponding to non-compacted myocardium. A mismatch between ${ }^{201} \mathbf{T 1}$ and BMIPP uptake in the area of noncompaction observed before carvedilol disappeared after treatment. Impaired sympathetic neuronal function shown by MIBG recovered after treatment. Thus carvedilol had beneficial effects on left ventricular function, hypertrophy, and both metabolic and adrenergic abnormalities in isolated left ventricular non-compaction. (Heart 2001;86:e4)
\end{abstract}

Keywords: isolated left ventricular non-compaction; carvedilol; cardiac sympathetic nerve; ventricular remodelling

Isolated left ventricular non-compaction is a rare disorder characterised by prominent trabeculations and deep intertrabecular recesses. ${ }^{1}$ The pathogenesis of this disorder is believed to be an arrest in endomyocardial morphogenesis. ${ }^{1}$ Prognosis is apparently grim, with a high mortality and morbidity from heart failure, ventricular arrhythmia, and systemic embolisation. ${ }^{12}$ Despite an increasing awareness of and interest in this disorder, there is still no information about the effects of medical treatment. This is the first report showing beneficial effects of carvedilol, a non-selective $\beta$ blocker, on global and regional left ventricular function, left ventricular mass, and scintigraphic findings in a patient with isolated left ventricular non-compaction.

\section{Case report}

A four month male infant was admitted to our institution for evaluation of congestive heart failure. He presented with a gallop rhythm and an enlarged heart on chest $x$ ray, with a cardiothoracic ratio of 0.68 . His ECG showed ST-T segment depression and an inverted T wave in the left precordial leads from V4-V7, without an abnormal $Q$ wave. On cross sectional echocardiography there was an enlarged left atrium and ventricle and a poorly contracting left ventricle with a fractional shortening of 0.12 . Characteristic echocardiographic features compatible with noncompacted myocardium ${ }^{1}$ were observed in apical, anterior, lateral, and inferior segments of the left ventricle but not the basal myocardium (fig 1).

Drug treatment was started initially with diuretics, digitalis, and an angiotensin converting enzyme inhibitor. Carvedilol was added subsequently, initially at a dose of $0.05 \mathrm{mg} / \mathrm{kg} /$ day orally. The dose was increased by $0.05 \mathrm{mg} /$ $\mathrm{kg} /$ day every week until it reached $0.2 \mathrm{mg} / \mathrm{kg} /$ day, and then by $0.2 \mathrm{mg} / \mathrm{kg} /$ day at monthly intervals up to $0.8 \mathrm{mg} / \mathrm{kg} /$ day. Fixed dose maintenance was continued for a further 14 months. There were no signs and symptoms of worsening heart failure, hypotension, or other adverse effects related to carvedilol during this period.

Cardiac catheterisation excluded coexisting cardiac and coronary anomalies. In addition, there was no family history of noncompaction, ${ }^{1}$ no facial dysmorphism, ${ }^{1}$ no ventricular thrombi, ${ }^{2}$ and no ventricular tachycardia ${ }^{2}$ complete bundle branch block, ${ }^{2}$ or Wolf-Parkinson-White syndrome. ${ }^{2}$

Findings before and 14 months after carvedilol treatment are summarised in table 1. The left ventricular ejection fraction increased from $30 \%$ to $57 \%$ during carvedilol treatment. Left ventricular end diastolic volume, end systolic volume, and end diastolic pressure decreased strikingly after treatment. After
Correspondence to: pkondou@pop02.odn.ne.jp

Accepted 31 January 2001 
A
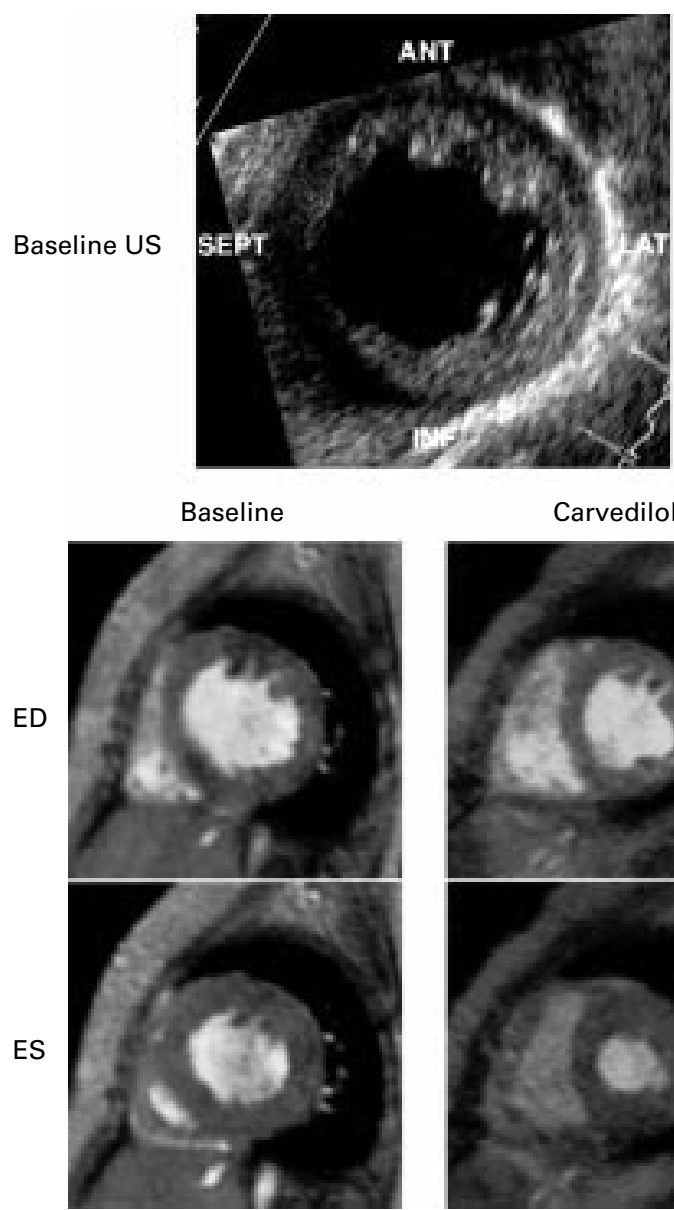

MIBG

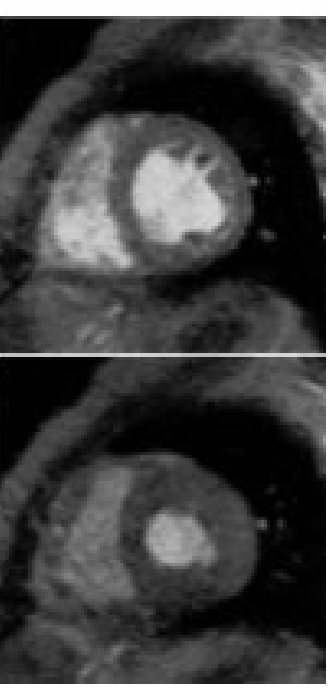

Early

Baseline

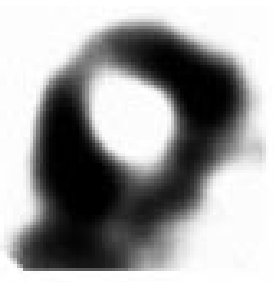

Delayed

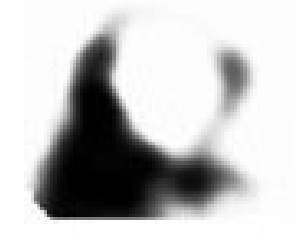

TI

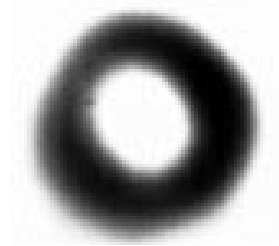

BMIPP

Figure 1 (A) top: a short axial echocardiogram of the left ventricle at the mid-ventricular level before carvedilol treatment, showing an echogenic subendocardial layer - which corresponds to non-compacted myocardium-in the anterior, lateral, and inferior walls. ANT, anterior wall; LAT, lateral wall; INF, inferior wall; SEPT, septum; US, ultrasound. (A) bottom: short axial mid-ventricular images of cine magnetic resonance imaging at end diastole (ED) and end systole (ES) before (left half) and after (right half) carvedilol treatment. Wall motion and myocardial thickening improved after treatment. (B) Short axial mid-ventricular images of single photon emission computed tomography with MIBG, Tl, and BMIPP before (left half) and after (right half) carvedilol treatment. Top half: before treatment, the MIBG image 15 minutes after injection (early) shows nearly normal uptake, while four hours later (delayed) anterior, lateral, and inferior walls show apparently decreased uptake, indicating preserved sympathetic innervation but impaired intravesicular retention of MIBG; after treatment, the abnormally accelerated washout of MIBG disappeared. Bottom half: before carvedilol treatment the BMIPP image shows relatively decreased uptake at anterior, lateral, and inferior walls compared with myocardial perfusion on the Tl image; after treatment, the mismatch between Tl and BMIPP findings disappeared. BMIPP, $\beta$-methyliodophenyl pentadecanoic acid; MIBG, metaiodobenzylguanidine.

carvedilol, non-compacted myocardium occupied a smaller percentage of the total ventricular wall area than before treatment.

We also performed magnetic resonance imaging before and after carvedilol (fig 1), and measured left ventricular mass and per cent wall thickening (table 1). Left ventricular mass decreased to about two thirds of the baseline value after treatment. Per cent wall thickening increased after carvedilol treatment in the segments corresponding to non-compacted myocardium.

Nuclear studies were performed using single photon emission computed tomography with ${ }^{201} \mathrm{Tl},{ }^{123} \mathrm{I}-\beta$-methyliodophenyl pentadecanoic acid (BMIPP), and ${ }^{123}$ I-metaiodobenzylguanidine (MIBG) (fig 1). Before carvedilol, there was a mismatch between ${ }^{201} \mathrm{Tl}$ and BMIPP uptake, indicating preserved myocardial viability but compromised oxidative fatty acid metabolism ${ }^{3}$ in the areas of noncompaction. Sympathetic nerve dysfunction in non-compacted areas was documented using
MIBG - a radiolabelled noradrenaline (norepinephrine) analogue taken up by the sympathetic neuronal terminals-before carvedilol treatment (fig 1). Nearly normal uptake 15 minutes after the injection of MIBG (MIBG early) and an obviously decreased uptake four hours later (MIBG delayed) indicated preserved cardiac innervation but impaired neuronal function (reduced ability to store MIBG in presynaptic vesicles ${ }^{4}$ ). After carvedilol treatment there was no longer a mismatch between ${ }^{201} \mathrm{Tl}$ and BMIPP, and recovery of neuronal function was demonstrated on MIBG delayed images.

\section{Discussion}

The main finding in this report was that carvedilol caused a major improvement in isolated left ventricular non-compaction, with beneficial effects on left ventricular function, mass, and scintigraphic findings. The mechanism of these effects is not yet clear because the cause of myocardial failure associated with ventricular non-compaction is not understood. 
Table 1 Demographic, haemodynamic, and scintigraphic variables in a case of isolated left ventricular non-compaction

\begin{tabular}{|c|c|c|c|}
\hline Variable & Baseline & After carvedilol & $p$ Value \\
\hline $\begin{array}{l}\text { Age (years) } \\
\text { Weight }(\mathrm{kg}) \\
\text { BSA }\left(\mathrm{m}^{2}\right)\end{array}$ & $\begin{array}{l}0.33 \\
6.3 \\
0.31\end{array}$ & $\begin{array}{l}1.83 \\
9.6 \\
0.44\end{array}$ & \\
\hline $\begin{array}{l}\text { LVEF }(\%) \\
\text { LVEDV (ml) } \\
\text { LVESV (ml) } \\
\text { LVEDP (mm Hg) } \\
\text { Mean PAP (mm Hg) } \\
\text { LV mass (g) } \\
\text { LV mass to EDV ratio }\end{array}$ & $\begin{array}{l}30 \\
47(347 \%) \\
33 \\
13 \\
18 \\
59.1 \\
1.26\end{array}$ & $\begin{array}{l}57 \\
28(126 \%) \\
12 \\
8 \\
12 \\
37.3 \\
1.33\end{array}$ & \\
\hline $\begin{array}{l}\text { Mean (SD) area of non-compaction } \\
(\%) \dagger\end{array}$ & $64.2(17.4)$ & $47.3(11.0)$ & 0.0598 \\
\hline $\begin{array}{l}\text { Mean (SD) LV wall thickening (\%)‡ } \\
\text { Septum } \\
\text { Anterior wall } \\
\text { Lateral wall } \\
\text { Inferior wall }\end{array}$ & $\begin{array}{l}27.0(11.0) \\
0.8(15.0) \\
1.9(12.8) \\
6.5(17.0)\end{array}$ & $\begin{array}{l}30.5(4.8) \\
29.1(24.8) \\
32.7(28.7) \\
59.2(32.1)\end{array}$ & $\begin{array}{l}\text { NS } \\
<0.01 \\
<0.01 \\
<0.01\end{array}$ \\
\hline $\begin{array}{l}\text { Myocardial tracer uptake (lateral/sept } \\
{ }^{123} \mathrm{I}-\mathrm{MIBG} \text { delayed } \\
{ }^{201} \mathrm{~T} 1 \\
{ }^{123} \mathrm{I}-\mathrm{BMIPP}\end{array}$ & $\begin{array}{l}0.68 \\
1.06 \\
0.89\end{array}$ & $\begin{array}{l}1.2 \\
1.34 \\
1.12\end{array}$ & \\
\hline $\begin{array}{l}{ }^{123} \text { I-MIBG washout rate }(\%) \S \\
\text { Septum } \\
\text { Lateral wall }\end{array}$ & $\begin{array}{l}25 \\
51\end{array}$ & $\begin{array}{l}19 \\
23\end{array}$ & \\
\hline
\end{tabular}

*Per cent of normal values in parentheses.

tMean values of per cent area of non-compaction of total ventricular wall in apical, mid-ventricular, and basal short axis echocardiographic images at end diastole and end systole.

$\ddagger$ Per cent wall thickening defined as [wall thickness at systole minus wall thickness at diastole] $/[$ wall thickness at diastole] $\times 100$. Average values of measurements at four points in each segment are shown.

\MIBG washout rate defined as [MIBG early uptake minus MIBG delayed uptake]/[MIBG early uptake] $\times 100$.

BSA, body surface area; ${ }^{123} \mathrm{I}$-BMIPP, ${ }^{123} \mathrm{I}-\beta$-methyliodophenylpentadecanoic acid; ${ }^{123} \mathrm{I}$-MIBG,${ }^{123} \mathrm{I}-$ metaiodobenzylguanidine; LV, left ventricular; LVEDP, left ventricular end diastolic pressure; LVEDV, left ventricular end diastolic volume; LVEF, left ventricular ejection fraction; LVESV, left ventricular end systolic volume; PAP, pulmonary artery pressure.

A previous report indicated that hypertrophic non-compacted segments are perfused from epicardial coronary arteries, which have no continuity with deep vascular communications with the left ventricular cavity. ${ }^{1}$ Microscopic necroses in the subendocardial area and within trabeculations have been documented in histological specimens, and these may be a result of ischaemia. ${ }^{2}$ Indeed it has been suggested that the myocardial failure and ventricular remodelling that occur in isolated left ventricular noncompaction are caused by ischaemia. ${ }^{2}$ Our finding of impaired aerobic fatty acid metabolism in non-compacted segments supports this possible pathogenesis.

Carvedilol reversed ventricular dilatation and hypertrophy in our patient. This effect of carvedilol in reversing remodelling has been widely recognised in myocardial infarction ${ }^{7}$ and idiopathic dilated cardiomyopathy. ${ }^{8}$ Interestingly, the reversal of remodelling seemed to occur preferentially in non-compacted rather than in compacted myocardium in the present study.

Another significant finding in our study relates to myocardial sympathetic dysfunction and its changes before and after carvedilol treatment. Sympathetic dysfunction has been observed in the myocardium under experimental remodelling. ${ }^{9}$ Carvedilol has an effect of reducing the abnormal release of noradrenaline from remodelling myocardium, ${ }^{8}$ and this may lead to myocardial contractile recovery, as shown in the present case. Of note, sympathetic dysfunction in viable myocardium has been recognised as a substrate for ventricular tachycardia, ${ }^{10}$ which is often associated with isolated left ventricular non-compaction. ${ }^{2}$

CONCLUSIONS

Carvedilol improved left ventricular function, hypertrophy, and both metabolic and adrenergic abnormalities in isolated left ventricular non-compaction, and this may result in an improved prognosis.

1 Chin TK, Perloff JK, Williams RG, et al. Isolated noncompaction of left ventricular myocardium. A study of eight cases. Circulation 1990;82:507-13.

2 Oechslin EN, Attenhofer Jost CH, Rojas JR, et al. Long-term follow-up of 34 adults with isolated left ventricular noncompaction: a distinct cardiomyopathy with poor prognosis. I Am Coll Cardiol 2000;36:493-500.

3 Knapp F, Franken P, Kropp J. Cardiac SPECT with iodine123-labeled fatty acids: evaluation of myocardial viability with BMIPP. F Nucl Med 1995;36:1022-30.

4 Henderson EB, Kahn JK, Corbett JR, et al. Abnormal I-123 metaiodobenzylguanidine myocardial washout and distribution may reflect myocardial adrenergic derangement in patients with congestive cardiomyopathy. Circulation 1988; 78:1192-9.

5 Nakajo M, Shimabukuro K, Yoshimura H. Iodine-131 metaiodobenzylguanidine intra- and extravesicular accumetaiodobenzylguanidine intra- and extravesicular
mulation in the rat heart. 7 Nucl Med 1986;27:84-9.

6 Junga G, Kneifel S, Von Smelal A, et al. Myocardial ischaemia in children with isolated ventricular non-compaction. Eur Heart f 1999;20:910-16.

7 Doughty RN, Whalley GA, Gramble G, et al. Left ventricular remodeling with carvedilol in patients with congestive heart failure due to ischemic heart disease. $₹ \mathrm{Am}$ Coll Cardiol 1997;29:1060-6.

8 Fujimura M, Yasumura Y, Ishida Y, et al. Improvement in left ventricular function in response to carvedilol is accompanied by attenuation of neurohumoral activation in patients with dilated cardiomyopathy. I Cardiac Failure 2000;6:3-10.

9 Kramer CM, Nicol PD, Rogers WJ, et al. Reduced sympathetic innervation underlies adjacent noninfarcted region dysfunction during left ventricular remodeling. $f$ Am Coll Cardiol 1997;30:1079-85.

10 Mitrani RD, Klein LS, Miles WM, et al. Regional cardiac sympathetic denervation in patients with ventricular tachycardia in the absence of coronary artery disease. $7 \mathrm{Am}$ Coll Cardiol 1993;22:1344-53. 